\title{
Finding the Elusive Sliding Phase in the Superfluid-Normal Phase Transition Smeared by $c$-Axis Disorder
}

\author{
David Pekker, ${ }^{1}$ Gil Refael, ${ }^{2}$ and Eugene Demler ${ }^{1}$ \\ ${ }^{1}$ Physics Department, Harvard University, 17 Oxford Street, Cambridge, Massachusetts 02138, USA \\ ${ }^{2}$ Physics Department, California Institute of Technology, MC 114-36, 1200 E. California Blvd., Pasadena, California 91125 , USA
}

(Received 2 April 2010; published 20 August 2010)

\begin{abstract}
We consider a stack of weakly Josephson coupled superfluid layers with $c$-axis disorder in the form of random superfluid stiffnesses and vortex fugacities in each layer as well as random interlayer coupling strengths. In the absence of disorder this system has a 3D $X Y$ type superfluid-normal phase transition as a function of temperature. We develop a functional renormalization group to treat the effects of disorder, and demonstrate that the disorder results in the smearing of the superfluid-normal phase transition via the formation of a Griffiths phase. Remarkably, in the Griffiths phase, the emergent power-law distribution of the interlayer couplings gives rise to a sliding Griffiths superfluid, with a finite stiffness in the $a-b$ direction along the layers, and a vanishing stiffness perpendicular to it.
\end{abstract}

PACS numbers: $67.85 . \mathrm{Hj}$

The interplay of disorder and broken symmetry remains a challenging and relevant problem for correlated quantum systems. The effects of disorder in one dimension, where the effects of quantum fluctuations are enhanced, is most dramatic, giving rise to Anderson localization [1], Dyson singularities, and random singlet phases [2]. Recent studies, both experimental and theoretical, concentrated on the superfluid-insulator transition of Bosonic chains [3], and strongly argued that disorder alters the universality of that transition [4]. While uncorrelated disorder in higher dimensions has a lessened effect, we must raise the question: how does correlated disorder, which only varies in a subset of directions, affect thermal and quantum phase transitions in higher dimensions?

Here, we study this question by concentrating on the superfluid-insulator transition in 3D Bose gases split into a series of pancake clouds by a 1D optical lattice with disorder, which varies only along the lattice direction. While this question is of much theoretical, and recently also experimental interest, it has not been addressed thus far. The effects of the disorder could be as mundane as just shifting the transition point, or as important as producing a new universality class of the transition or obliterating it altogether. Indeed, we shall show that the interplay between disorder along the $c$ axis and the $a-b$ plane Berezinskii-Kosterlitz-Thouless (BKT) physics [5,6], smears the transition, giving rise to an intermediate Griffiths phase [7,8] that occupies a wide region of the phase diagram. This disorder induced intermediate phase is distinct from the superficially similar intermediate phase found in superfluid bilayers that arises from the interplay of coupling constants [9]. Furthermore, in part of the Griffiths phase, the superfluid becomes split into an array of 2D puddles with no stiffness within this Griffiths phase, the superfluid becomes split into an array of $2 \mathrm{D}$ puddles that have no stiffness along the $c$ axis, thus realizing the illusive sliding phase paradigm [10], supporting superflow only in the $a$ and $b$, but not the $c$ directions.

The questions we raise are fast becoming important for experiments. Experiments on ultracold atoms observed both the BKT transition in large 2D "pancakes" produced by very deep 1D optical lattices [11], and Anderson localization of Bosons in 1D disordered optical lattices $[3,12]$. Our system can be realized by constructing a stack of large 2D pancakes using a disordered 1D optical lattice, and tests the effects of disorder near the 2D-3D crossover [13,14].

The model which we analyze consists of a set of coupled 2D superfluid layers. Each layer has a superfluid stiffness $K_{m}$, vortex fugacity (akin to vortex density per coherence length) $\zeta_{m}$, and Josephson coupling (to the next layer) $J_{m}$. $K_{m}, \zeta_{m}$, and $J_{m}$ are initially random and uncorrelated [15] [Fig. 1(a)]. Our analysis combines a Kosterlitz-Thoulesslike momentum space RG for the in-plane degrees of freedom $[6,16]$ with a real-space $\mathrm{RG}[2,4]$. In the realspace RG step, strongly coupled layers $\left(J_{m} \sim 1\right)$ are merged, while vortex-ridden layers $\left(\zeta_{m} \sim 1\right)$ are considered normal and are perturbatively eliminated.

Let us first summarize the phase diagram we find [Fig. 1(b) and Table I]. At low temperatures the system forms a 3D superfluid. As the temperature is raised, a Griffiths phase appears; in it, the system breaks up into 2D superfluid puddles, each composed of one or several pancakes, with weak (power-law distributed) interpuddle tunneling. At yet higher temperatures increased further, the $c$-axis superfluid response disappears, while the system remains superfluid in the $a$ and $b$ directions, realizing a sliding phase. At the highest temperatures, the in-plane superfluid response smoothly vanishes as the system becomes fully normal.

The power-law distributions that characterize the Griffiths phase are a direct consequence of the disorder. At intermediate temperatures, many layers have strong fluctuations and become incoherent. The remaining layers, 


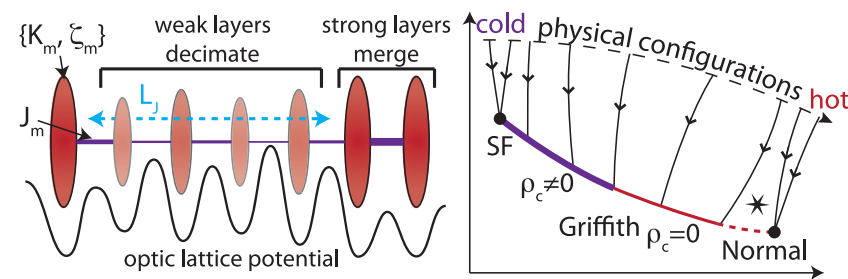

FIG. 1 (color online). Left: Schematic diagram of the model: red ovals (purple bars) represent the superfluid layers (Josephson couplings) with size inversely proportional to vortex fugacity $\zeta_{m}$ (directly proportional to Josephson coupling $J_{m}$ ). The effects of the real-space RG is to merge strong layers, and decimate weak layers. Emergent length-scale $L_{J}$ corresponds to the typical separation between strong layers. Right: Schematic diagram of the RG flows showing the superfluid (SF) and normal fixed points along with the Griffiths fixed line. The black dashed line represents physical configurations, with points to the right corresponding to higher temperatures. The Griffiths fixed line is split into two segments, corresponding to the regimes with finite and zero $c$-axis superfluid response. The star indicates a possible unstable fixed point [24].

on either side of an incohernet layer, can still exchange bosons but with an attenuated amplitude, e.g., $J_{\text {eff }}=$ $J_{m-1} J_{m}$ if layer $m$ is eliminated. On average, a pair of coherent layers are separated by $L_{J}$ incoherent ones Fig. 1(a). The elimination of the incoherent layers gives rise to the power-law distribution $P(J) \sim J^{\nu_{J}-1}$ of interlayer tunnelings $J_{m}$. $L_{J}$ determines $\nu_{J}: \nu_{J} \sim \log [1 / \bar{J}] / L_{J}$, with $\bar{J}$ the typical initial Josephson coupling.

The Griffiths phase can be separated into two regimes. A sliding regime with $\nu_{J}$ flowing to $\nu_{J}<1$ [Fig. 1(b)], and no $c$-axis stiffness, and a Griffiths superfluid with a finite $c$-axis stiffness and $\nu_{J}>1$. Both regimes have a vanishingly small $c$-axis critical current. To wit, the critical current of $n$ layers is determined by the weakest effective tunneling. The expectation value for the longest run of weak layers is $\mathcal{R}_{n} \sim \log _{1 / p_{w}}\left[n\left(1-p_{w}\right)\right]$ [17] (with $p_{\text {weak }}$ the probability of a layer to be normal in the first epoch; $\left.L_{J}=\left(1-p_{w}\right)^{-1} \gg 1\right)$. Thus $I_{c} \sim\left(\frac{n}{L_{J}}\right)^{L_{J} \log \bar{J}}$.

Model.-Our model consists of a stack of coupled $1+1$ dimensional (Euclidean) sine-Gordon models [16] with partition function $Z=\operatorname{Tr} \exp -\sum_{m}\left(S_{s G ; m}+S_{J ; m, m+1}\right)$ where

$$
\begin{array}{r}
S_{s G ; m}=\int d y d x\left[K_{m}\left(\partial_{x} \theta_{m}\right)^{2}+\frac{1}{K_{m}}\left(\partial_{x} \phi_{m}\right)^{2}\right. \\
\left.-2 i\left(\partial_{x} \phi_{m}\right)\left(\partial_{y} \theta_{m}\right)+\zeta_{m} \cos \left(2 \phi_{m}\right)\right], \\
S_{J ; m, m+1}=\int d y d x J_{m} \cos \left(\theta_{m}-\theta_{m+1}\right) .
\end{array}
$$

Here, the index $m$ is the vertical position of the layer in the stack, $S_{s G ; m}$ is the sine-Gordon action describing the density waves and vortices; $S_{J ; m, m+1}$ is the $m$ to $m+1$ tunneling; $\theta_{m}(x, y)$ and $\phi_{m}(x, y)$ are the superfluid orderparameter phase variable and its conjugate, respectively, $\left(\left[\theta_{m}(r), \partial_{x} \phi_{m^{\prime}}\left(r^{\prime}\right)\right]=i \pi \delta\left(r-r^{\prime}\right) \delta_{m, m^{\prime}}\right)$. We define $J_{m}=$
TABLE I. Properties of the various phases.

\begin{tabular}{lccccc}
\hline \hline$T$ & phase & $\rho_{a b}$ & $\rho_{c}$ & $J_{c, a b}$ & $J_{c, c}$ \\
\hline high & normal & zero & zero & zero & zero \\
& Griffith-sliding Griffiths & finite & zero finite & finite & zero \\
low & superfluid & finite & finite & finite & finite \\
\hline \hline
\end{tabular}

$\mathcal{J}_{m} / T, K_{m}=\mathcal{K}_{m} / T$, and $\zeta_{m} \sim e^{\left(-E_{\text {core }, m} / T\right)}$ as the reduced Josephson coupling, superfluid stiffness, and vortex fugacity at temperature $T$, where $E_{\text {core }, m}$ is the vortex core energy. The sine-Gordon model requires a short-distance cut-off, which we choose as $a$ for all layers, and use units where $a=1$. Without disorder, this model exhibits a dimensional crossover from 2D-like to 3D-like behavior, followed by a conventional 3D $X Y$ superfluid-to-normal transition [18].

Renormalization group.-Our analysis relies on a combined $c$-axis real space and $a$ - $b$ momentum space RG. The momentum space transformation is given by [19]

$$
\begin{gathered}
\frac{d J_{m}}{d \ell}=\frac{J_{m}}{4 \pi}\left[8 \pi-\frac{1}{K_{m}}-\frac{1}{K_{m+1}}\right]-\frac{\pi^{2}}{2} J_{m}\left(\zeta_{m}^{2}+\zeta_{m+1}^{2}\right), \\
\frac{d \zeta_{m}}{d \ell}=\zeta_{m}\left[2-\pi K_{m}\right]-\frac{1}{2} \pi^{2} \zeta_{m}\left(J_{m}^{2}+J_{m-1}^{2}\right), \\
\frac{d K_{m}}{d \ell}=-2 \pi^{3}\left(K_{m} \zeta_{m}\right)^{2}+\frac{\pi}{2}\left(J_{m}^{2}+J_{m-1}^{2}\right) .
\end{gathered}
$$

To lowest order in $\zeta_{m}$ and $J_{m}$, there is a range of superfluid stiffnesses $1 / 4 \pi \lessgtr K_{m} \lesssim 2 / \pi$ in which both the vortex fugacity $\zeta_{m}$ and the Josephson coupling $J_{m}$ are relevant and their competition gives rise to the Griffiths phase. Outside this range the system is strongly superfluid (large $K_{m}$ ) or strongly insulating (small $K_{m}$ ), Fig. 2 .

As the in-plane momentum shell RG proceeds, the realspace RG (RSRG) merges or eliminates layers. When a Josephson coupling becomes large, $J_{i}=1$, the relative phase $\Delta \theta=\theta_{i+1}-\theta_{i}$ of the two neighboring layers becomes locked and the two layers merge into a superlayer having $K_{\text {eff }}=K_{i}+K_{i+1}$ and $\zeta_{\text {eff }}=\zeta_{i} \zeta_{i+1}$. Similarly, if a vortex fugacity becomes large, $\zeta_{i}=1$, then the conjugate field $\phi_{i}$ in that layer becomes locked and vortices proliferate. Upon integrating out the incoherent layer, we find that it suppresses tunneling across it to $J_{\text {eff }}=J_{i-1} J_{i}$. These RG rules make it convenient to parametrize $J$ and $\zeta$ in terms of their $\operatorname{logs} j=\log (1 / J), z=\log (1 / \zeta)$.

In lieu of a numerical analysis [20] of the RG flow, let us derive the approximate flow of the distributions of $K, j$, and

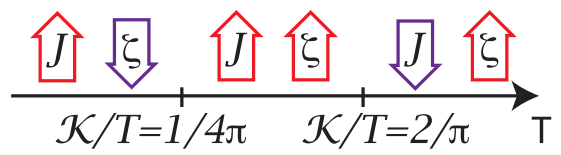

FIG. 2 (color online). Schematic: relevance of $J$ and $\zeta$ as a function of temperature. 
$z$, and their universal aspects. First, note that the RSRG layer merging step leads to strong correlations of $K$ 's and $\zeta$ 's. Therefore, alongside the distribution $P_{j}$ for $j_{m}$ 's, we use the joint probability distribution $Q_{K}^{z}$ for $z_{m}$ 's and $K_{m}$ 's. The functional RG (fRG) equations resulting from Eqs. (3) and (4) are

$$
\begin{aligned}
\frac{d P_{j}}{d \ell}= & I_{1} \partial_{j} P_{j}-\int d K_{1} Q_{K_{1}}^{0}\left\{2-\pi K_{1}\right\} P_{j} \\
& +\int d K_{1} Q_{K_{1}}^{0}\left\{2-\pi K_{1}\right\} \int d j^{\prime} P_{j^{\prime}} P_{j-j^{\prime}}+I_{1} P_{0} P_{j},
\end{aligned}
$$

$$
\begin{aligned}
\frac{d Q_{K}^{z}}{d \ell}= & (2-\pi K) \partial_{z} Q_{K}^{z}-\int d 1 Q_{1}\left(2-\frac{1}{4 \pi K_{1}}-\frac{1}{4 \pi K}\right) Q_{K}^{z} P_{0} \\
& +\int d 1 Q_{1} Q_{K-K_{1}}^{z-z_{1}}\left(2-\frac{1}{4 \pi K_{1}}-\frac{1}{4 \pi\left(K-K_{1}\right)}\right) P_{0} \\
& +\int d K_{1} Q_{K_{1}}^{0}\left\{2-\pi K_{1}\right\} Q_{K}^{z},
\end{aligned}
$$

where $I_{1}=\int d 1 d 2 Q_{1} Q_{2}\left(2-\frac{1}{4 \pi K_{1}}-\frac{1}{4 \pi K_{2}}\right),\{g\}$ stands for $g \Theta(g)$ with $\Theta$ being the step function; $d 1$ and $Q_{1}$ are shorthand for $d K_{1} d z_{1}$ and $Q_{K_{1}}^{z_{1}}$ where it is unambiguous. Briefly, the first terms of Eqs. (6) and (7) correspond to the action of the linear in $J_{m}$ and $\zeta_{m}$ terms of the momentum space RG Eqs. (3) and (4). The remaining terms correspond to the action of the real-space RG, and the rescaling of $P_{j}$ and $Q_{K}^{z}$ whenever layers are removed from the system to maintain normalization. The fRG equations must be supplemented by absorbing wall boundary conditions $Q_{K}^{0^{-}}=0$ and $P_{0^{-}}=0$ which remove the small $z$ 's and $j$ 's (large $\zeta$ 's and $J$ 's) from the distributions when layers are decimated or merged. In order to compute physical observables we also keep track of $n(\ell)$, the number of surviving layers at the RG scale $\ell$ :

$$
\frac{d n}{d \ell}=-n\left(I_{1} P_{0}+\int d K_{1}\left\{2-\pi K_{1}\right\} Q_{K_{1}}^{0}\right)
$$

The structure of the fRG is similar to that of Refs. [8,21] for the damped transverse field Ising model [22].
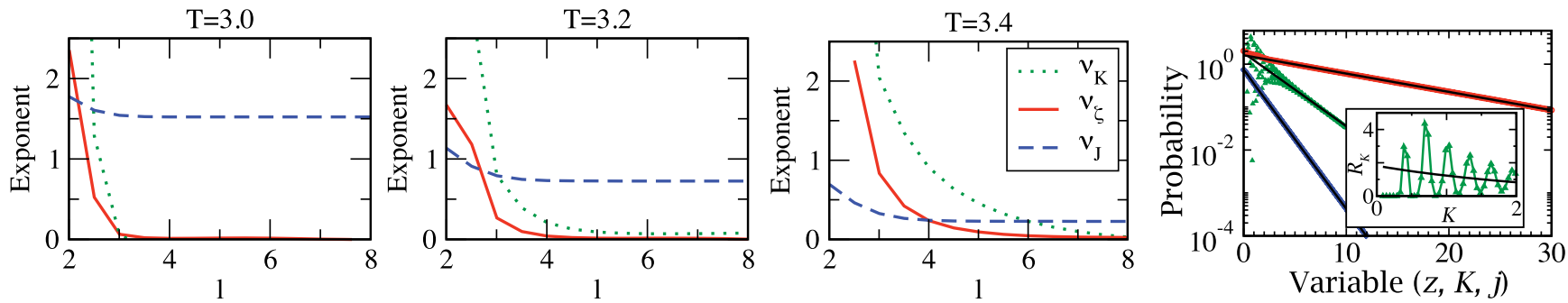

FIG. 3 (color online). Left three panels: flow of exponents $\nu_{K}, \nu_{\zeta}$, and $\nu_{J}$ under the action of RG for three different initial distributions corresponding to temperatures $T=3.0,3.2$, and 3.4. $\ell \sim 3$ separates the first and second epochs. The asymptotic value of $\nu_{J}$ at large length-scales indicates that $T=3.2$ and 3.4 correspond to the sliding regime. Right panel: semilog plot of typical distributions (from top to bottom) $Q[z], R[K]$, and $P[j]$, obtained by solving Eqs. (6) and (7) numerically and the corresponding exponential fits (black solid lines) that are used to obtain the values of exponents $\nu_{\zeta}, \nu_{K}$, and $\nu_{J}$. The inset depicts the distribution $R[K]$ on a linear-linear plot, the oscillations arise from additions of $K$ values when layers merge.

085302-3 


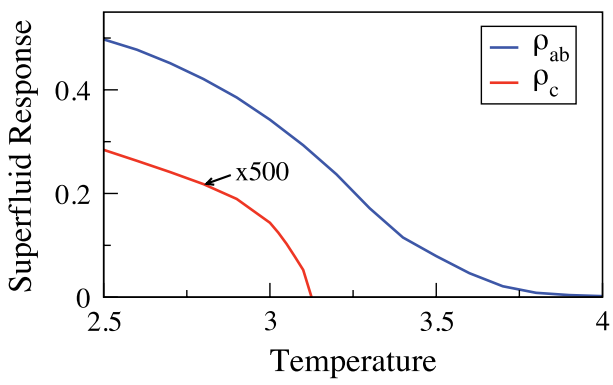

FIG. 4 (color online). In-plane $\left(\rho_{a b}\right)$ and out-of-plane $\left(\rho_{c}\right)$ stiffness as a functions of temperature (evaluated at $\ell=10$ ).

As $\rho_{c}$ depends on the area, we include the factor of $e^{-2 l}$ in Eq. (10) to account for its renormalization. We plot $\rho_{a b}$ and $\rho_{c}$ vs $T$ near saturation (at large value of the RG parameter $\ell$ ) in Fig. 4. The smearing of the SF-N transition is reflected in $\rho_{a b}$ decreasing smoothly, as $T$ is increased, reaching zero at the end of the Griffiths fixed line (without following any power law). $\rho_{c}$ decreases much faster, becoming zero within the Griffith phase at the point where $\lim _{\ell \rightarrow \infty} \nu_{J}(\ell)=$ $1(T \sim 3.1)$. The disappearance of $\rho_{c}$ signals the onset of the elusive sliding subphase; see Table I.

Experimental probes.-In the setting of ultracold atoms, the superfluid response as well as the critical current could be measured by quickly displacing the trap potential and looking at the decay of the center of mass oscillations [25]. Alternatively, correlations can be measured via shot noise in interference experiments [20]. In the mesoscopic setting, the Griffiths phase could appear in artificially grown structures composed of alternating layers of superconducting and insulating films of varying thicknesses. In this setting superfluid responses and critical currents could be measured directly.

Conclusions.-The $c$-axis disorder we study is interesting as it is at the dimensional boundary between disorder being relevant and irrelevant, i.e., the transition being completely smeared by more correlated disorder or remaining sharp with less correlated disorder [7]. We have developed a functional renormalization group scheme to treat $c$-axis disorder. Using it, we show that, due to the BKT transition in individual layers, the system-wide transition is smeared into a Griffith phase. Further, within the Griffith phase the system becomes essentially two dimensional. The reduction of dimensionality is reflected in the strong anisotropy of physical observables like critical current and superfluid response.

It is our pleasure to thank Michael Lawler, Subir Sachdev, and Bryan Clark for insightful discussions. D. P. and E.D. acknowledge support from DARPA, CUA, and NSF Grant No. DMR-07-05472. G. R. gratefully acknowledges support from the Packard Foundation, the Sloan Foundation, and the Cottrell Scholars program.

Note added.-Recently, we became aware of a complementary investigation from a scaling perspective by Mohan, Goldbart, Narayanan, Toner, and Vojta [26], which is consistent with our findings.
[1] P. W. Anderson, Phys. Rev. 109, 1492 (1958).

[2] D. S. Fisher, Phys. Rev. B 50, 3799 (1994); D. S. Fisher, Phys. Rev. B 51, 6411 (1995).

[3] J. Billy, V. Josse, Z. Zuo, A. Bernard, B. Hambrecht, P. Lugan, D. Clement, L. Sanchez-Palencia, P. Bouyer, and A. Aspect, Nature (London) 453, 891 (2008); G. Roati, C. D’Errico, L. Fallani, M. Fattori, C. Fort, M. Zaccanti, G. Modugno, M. Modugno, and M. Inguscio, Nature (London) 453, 895 (2008).

[4] E. Altman, Y. Kafri, A. Polkovnikov, and G. Refael, Phys. Rev. Lett. 93, 150402 (2004); E. Altman, Y. Kafri, A. Polkovnikov, and G. Refael, Phys. Rev. Lett. 100, 170402 (2008).

[5] V. L. Berezinskii, Sov. Phys. JETP 34, 610 (1972).

[6] J. M. Kosterlitz and D. J. Thouless, J. Phys. C 6, 1181 (1973).

[7] T. Vojta, J. Phys. A 39, R143 (2006).

[8] J.A. Hoyos and T. Vojta, Phys. Rev. Lett. 100, 240601 (2008); T. Vojta, C. Kotabage, and J. A. Hoyos, Phys. Rev. B 79, 024401 (2009).

[9] H. A. Fertig, Phys. Rev. Lett. 89, 035703 (2002); W. Zhang and H. A. Fertig, Phys. Rev. B 71, 224514 (2005).

[10] C. S. O'Hern, T. C. Lubensky, and J. Toner, Phys. Rev. Lett. 83, 2745 (1999).

[11] Z. Hadzibabic, P. Kruger, M. Cheneau, B. Battelier, and J. Dalibard, Nature (London) 441, 1118 (2006).

[12] See, e.g., the review L. Fallani, C. Fort, and M. Inguscio, arXiv:0804.2888 and references therein.

[13] I. Affleck and B. Halperin, J. Phys. A 29, 2627 (1996).

[14] S. Burger, F. S. Cataliotti, C. Fort, P. Maddaloni, F. Minardi, and M. Inguscio, Europhys. Lett. 57, 1 (2002); W. Li, H.-C. Chien, and M. Kasevich (private communications).

[15] Throughout we assume bounded distributions, as otherwise the system would always become disconnected.

[16] M. A. Cazalilla, A. F. Ho, and T. Giamarchi, New J. Phys. 8, 158 (2006).

[17] M. F. Schilling, The College Math. J. 21, 196 (1990).

[18] I. Affleck and B. I. Halperin, J. Phys. A 29, 2627 (1996).

[19] These equations are similar to those of Ref. [16], but adapted to account for unequal coupling constants in neighboring layers.

[20] B. K. Clark, D. Pekker, G. Refael, and E. Demler (to be published).

[21] A. Del Maestro, B. Rosenow, M. Muller, and S. Sachdev, Phys. Rev. Lett. 101, 035701 (2008).

[22] An important difference is that previous treatments used an energy scale to drive the real-space RG, while we use momentum space $\mathrm{RG}$ to drive the real-space $\mathrm{RG}$.

[23] Qualitative results are independent of the particular choice of distributions as long as they are bounded.

[24] We suspect that there may be an additional unstable fixed point, indicated by the star in Fig. 1(b). However, due to the complexity of the fRG equations, we were unable to find it or rule it out.

[25] D. McKay, M. White, M. Pasienski, and B. DeMarco, Nature (London) 453, 76 (2008); M. Pasienski, D. McKay, M. White, and B. DeMarco, arXiv:0908.1182.

[26] P. Mohan, P. M. Goldbart, R. Narayanan, J. Toner, and T. Vojta, preceding Letter, Phys. Rev. Lett. 105, 085301 (2010). 